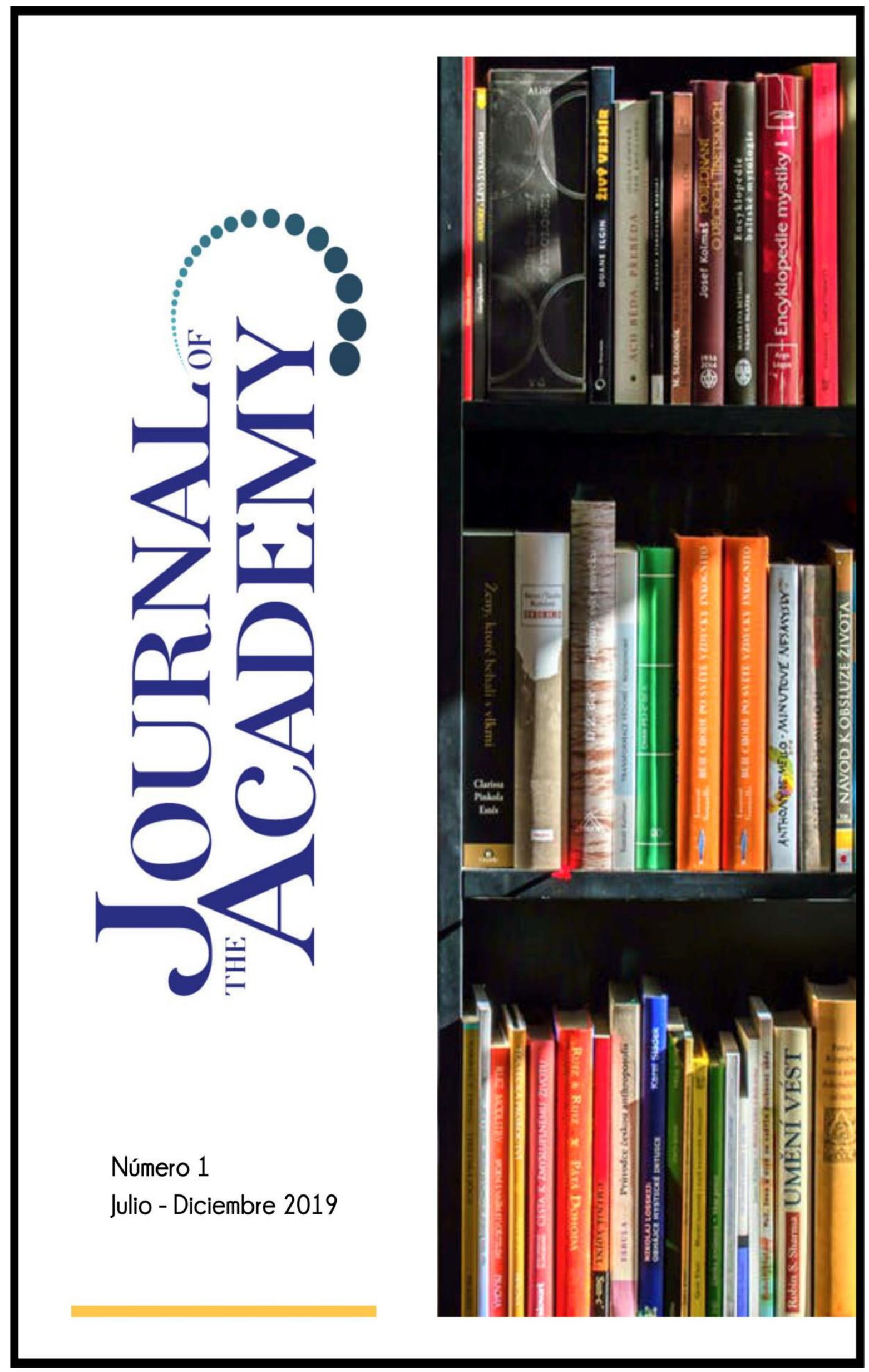




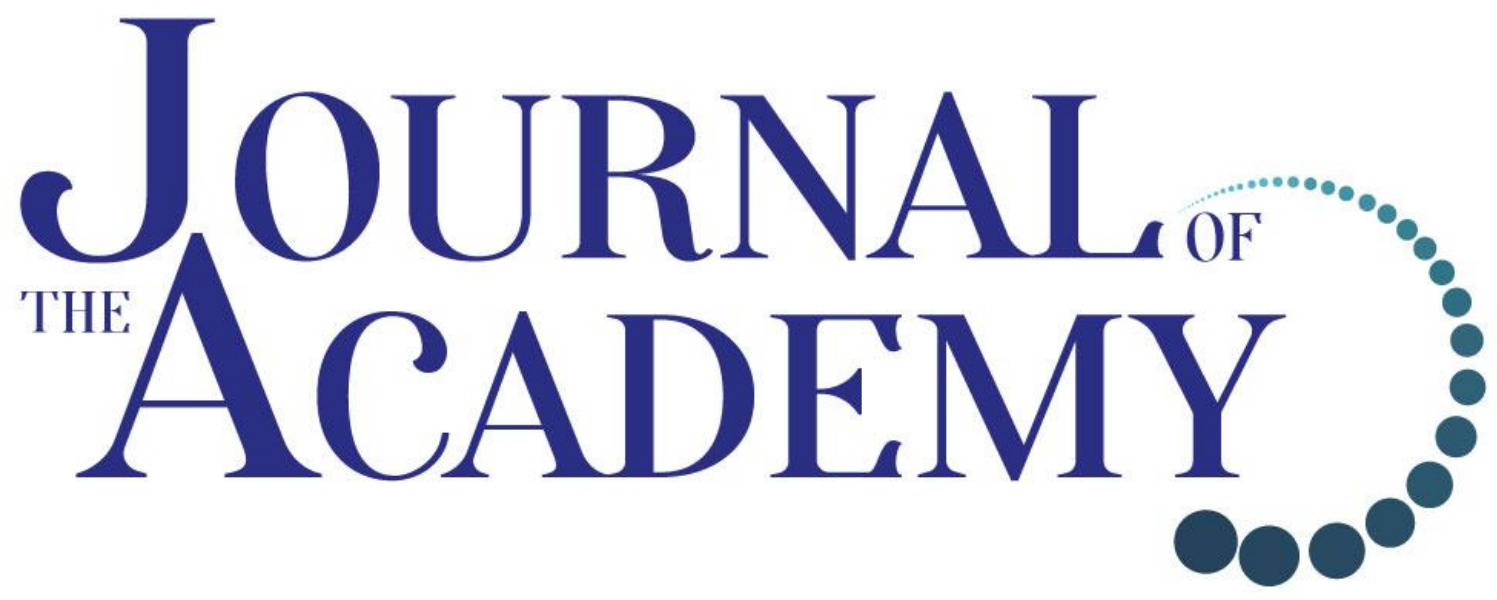

Publicación Científica de la Asociación de Universidades del Perú ASUP

Número 1

Julio-Diciembre 2019

www.journalacademy.org 


\section{ISSN 2707-0301}

\section{Directora-Editora en Jefe}

Ada Gallego Ruiz Conejo

Editor Científico

Oswaldo Orellana Manrique

Comité Científico

\section{Javier Carreón Guillén}

Universidad Nacional Autónoma de México, México Martino Contu

Universidad de Sassari, Italia

Jorge Enrique Elías Caro

Universidad del Magdalena, Colombia

Roberto Escalante Semerena

Universidad Nacional Autónoma de México, México

Oscar Ortega Arango

Universidad Autónoma de Yucatán, México

Alex Veliz Burgos

Universidad de Los Lagos, Chile

Comité Editorial

\section{Manuela Garau}

Centro Studi Sea, Italia

José Manuel González Freire

Universidad de Colima, México

Carlos Tulio da Silva Medeiros

Diálogos en Mercosur, Brasil

Eduardo Gomes Onofre

Universidade Estadual da Paraíba, Brasil

\section{Equipo Ejecutivo}

Juan Carlos Norabuena Castaneda

Thalia Chávez Cortéz

Journal Academy

Revista Semestral Open Access 


\title{
CONTROL INTERNO Y DESARROLLO LOCAL DE LAS MUNICIPALIDADES
}

\section{INTERNAL CONTROL AND LOCAL DEVELOPMENT OF MUNICIPALITIES}

Recibido: 22 de mayo de 2018

Aceptado: 04 de mayo de 2019

Edgar VELASQUEZ VASQUEZ

Universidad Andina del Cusco

evelasquezv@uandina.edu.pe

\begin{abstract}
This research article deals with the Internal Control and its correlation with the Local Development of the Municipalities of the Canchis Province 2018, in particular the objective of the research was to define how the local development of the municipalities of the province of Canchis and the internal control. The importance of the research lies in contrasting the influence of internal control on the local development of the municipalities of Canchis. The research is a non-experimental model, the corresponding research design is the descriptive correlational characterized by comprising the following steps: the data was collected for each variable to describe them according to their dimensions, for internal control: supervision, communication and information, risk assessment, control activities, risk assessment and control environment, for local development: environmental development, social development and economic development, a survey was applied to the sample constituted by 64 officials who work in the municipalities of the districts of the province of Canchis, the non-parametric statistic of Spearman's ratio factor was estimated and doing hypothesis docimasia it was concluded that there is very high linear correlation significant in the middle of the internal control and the local development of the municipalities the province of Canchis 2018.
\end{abstract}

Keywords: internal control, local development

Resumen: Este artículo de investigación trata del Control Interno y su correlación con el desarrollo Local de las Municipalidades de la Provincia de Canchis 2018, en particular el objetivo de la investigación fue definir cómo se relacionan el desarrollo local de los municipios de la provincia de Canchis y el control interno. La trascendencia de la investigación radico en contrastar el influjo del control interno en el desarrollo local de las municipalidades de Canchis. La investigación es de modelo no experimental, el diseño de investigación que corresponde es el descriptivo correlacional se caracteriza porque comprende los siguientes pasos: se recogió los datos respecto de cada variable para describirlos en función a sus dimensiones, para el control interno: supervisión, comunicación e información, evaluación de riesgos, actividades de control, evaluación de riesgos y ambiente de control, para el desarrollo local: desarrollo ambiental, desarrollo social y desarrollo económico, se aplicó una encuesta a la muestra constituida por 64 funcionarios que laboran en las municipios de los distritos de la provincia de Canchis, Se estimó el estadístico no paramétrico de factor de relación de Spearman y haciendo docimasia de hipótesis se llegó a concluir que hay correlación lineal muy alta significante en medio del control interno y el desarrollo local de los municipios la provincia de Canchis 2018.

Palabras claves: control interno, desarrollo local.

\section{Para Citar este Artículo:}

Velásquez Vásquez. Control interno y desarrollo local de las Municipalidades. Jornal of Academy num 1 (2019): 32-43.

Licencia Creative Commons Atributtion Nom-Comercial 3.0 Uunported

(CC BY-NC 3.0)

Licencia Internacional 


\section{Introducción}

En los municipios distritales de la provincia de Canchis departamento del Cusco cuentan con recursos humanos y económicos a disposición, tales recursos no están siendo bien canalizados ni utilizados; teniendo como causa a distintos factores: Desviación de políticas, metas y objetivos planteados por los municipios, no cumplimiento de los requisitos legales e internos, no información a la administración, estrategias inefectivas para la evaluación del Control Interno y Sistema de Gestión de Calidad, no exactitud en la comunicación, obsoleta consultoría, etcétera.

Por otra parte (Mamani, 2017), infiere que el control interno influye positivamente en la gestión administrativa para el cumplimiento de metas y objetivos institucionales, siempre y cuando se esté aplicando correctamente los sub componentes y componentes del control interno que establece el informe COSO (Comité de organizaciones patrocinadoras de la comisión Treadway). Al respecto, (Dugarte, 2012), añade que todos los elementos del control interno conforme al planteamiento del Informe COSO necesariamente deben considerarse para la determinación de patrones de control interno administrativo en realización de trabajos puesto que forman un conjunto integro de control mediante la interrelación de los todos los componentes generan directa relación puesto que los elementos se interrelacionan a las acciones operativas como una estructura verdadera de desarrollo y conforman los propósitos de los elementos de la gestión pública del municipio. Por otro lado, (Guevara, 2016), sugiere que, los funcionarios del municipio, dirigidos por su alcalde, implementen el sistema de control interno conforme a lo definido por reglas cosmopolitas (Informe COSO) y las reglas nacionales (Contralaría General de la República).

Se establece la influencia del Control Interno en la Gestión de los Gobiernos Locales de las Municipalidades (Crisologo, 2013). Según expresa Salazar, citado por (Soto, 2017), que el análisis del Control Interno implica la mejora de los procedimientos de administración del SAT (Sistema de Administración Tributaria). También (Acuña, 2013), sostiene que el control interno influye positivamente en la Unidad de abastecimiento del municipio. Por otra parte, (Condo, 2017), concluye se ha demostrado las causas de la implantación del control interno, que son: el desconocimiento de algunos funcionarios y trabajadores a la Implantación del Sistema de Control Interno; falta de actualización y capacitación a los funcionarios y trabajadores y personal idóneo en el tema; poco interés por parte de la gestión a la Implementación del Sistema de Control Interno y ausencia de disposición de recursos económicos para su implementación. Además (Martínez, M. y Del Carmen, 2016), concluye que los controles internos aplicados a la Dirección de proyectos son eficientes porque permiten desarrollar los proyectos en tiempo y forma según lo planificado y con su debido seguimiento. Además, es necesario considerar la conclusión a que llega (Melgarejo, 2016), en su trabajo de investigación, en la cual deduce la existencia de correlación significante entre el Control interno y la administración del municipio.

En relación al ambiente de control, este componente establece un ámbito organizacional en favor del ejercicio de conductas, valores, buenas prácticas y reglas convenientes, para concienciar a los componentes de la institución y generan una educación de control interno. Todos estos criterios apropiados coadyuvan al fortalecimiento y establecimiento de políticas y procedimientos de control interno que dirigen a la consecución de la cultura institucional de control y de los objetivos institucionales (Vizcarra, 2017). Al respecto, (Samaniego, 2013), señala que el Ambiente de Control repercute en el logro de metas y objetivos de la entidad. Po otra parte (Soto, 2017), sugiere que las municipalidades distritales deben contar con la existencia de documentos de gestión como evaluaciones de clima laboral, (Manrique, 2018), plantea al director de la municipalidad del tema de estudio, enmendar los aspectos relacionados a los elementos del ambiente de control, actividades de control y se recomienda a la autoridad y al gerente de la Municipalidad a mejorar la valoración de la estructura del control interno, teniendo por objetivo detectar y corregir sus defectos y mejorar constantemente sus reglas de control interno para que sean aplicadas a las acciones y relaciones de la entidad y susáreas. 
Respecto a la Evaluación de Riesgos, (Boza et al., 2013), propone la implementación de un Sistema de Gestión en salud, una guía que oriente su aplicación, y una herramienta que favorezca su evaluación dentro de la Municipalidad.

Así mismo se establece que el nivel de valoración de contingencia del control interno que incide es significativo en el mejoramiento de calidad de vida de los habitantes (Flórez, 2015); corrobora (Crisologo, 2013), estableciendo que el nivel de evaluación de riesgo influye en el mejoramiento de condición de vida de los habitantes. De acuerdo con (Ñañez, 2017), sugiere identificar y analizar los riesgos que pueda perjudicar a la entidad; a la vez realizar acciones de supervisión para prevenir errores, lograr objetivos adecuados y para el bien común.

En relación a las actividades de control, (Marin, 2016), considera como normas a: procedimientos de autorización y aprobación, segregación de funciones, evaluación costo- beneficio, controles sobre el acceso a los recursos y archivos, verificaciones y conciliaciones, rendición de cuentas, documentación de procesos, actividades y tareas, revisión de procesos, actividades y tareas y que estas se deben cumplir a cabalidad para un óptimo control interno en el desarrollo local del municipio. Al respecto, se determinó que si no se hace uso de las diversas clases de control interno en la ejecución de obras por gestión directa en las municipalidades, esto se observa en la relativa capacitación del personal, bajos grados de control, ausencia de claridad en las cuentas, sin ser previamente revisadas (Hurtado de la Cruz, 2015). Por otra parte, (Layme, 2014), señala que entre reglas y procesos de control interno, hay significancia estadística, o sea se propone las reglas y procesos básicos para la implantación de la estructura del Control Interno que mejoren sus acciones y relaciones, en la SPBS. Además, (Flórez, 2015), establece que los grados de acciones de control incurren en el presupuesto de gasto versus rendimiento de la ejecución presupuestal. Además (Acuña, 2013), señala la efectividad y el mejoramiento del Sistema de Control Interno en las acciones control y almacén de bienes patrimoniales del municipio.

Cuando se trata de comunicación municipal, cabe interrogarse por el nivel de democratización que se quiere lograr en la gestión, su repercusión en la población y en el desarrollo local. La comunicación municipal considera a la gestión local en su conjunto: sus áreas, estrategias, proyectos, fines, etc. Según (Flórez, 2015), señala que, por los productos conseguidos en la indagación, se determina que el nivel de comunicación e información incrementa en forma favorable los grados de eficacia, eficiencia y economía. Por consiguiente, hay una incidencia significante con relación a la administración del gobierno local de las municipalidades. Similarmente (Crisologo, 2013), corrobora que el nivel de comunicación e información implica el mejoramiento en favor de los niveles de economía, eficiencia y eficacia. Por otro lado, según (Zamata, 2016), una de las funciones del CCl es comunicar a todo el personal de la importancia de contar con un SCl. Por otra parte, según (Samaniego, 2013), la Información y Comunicación si inciden en la definición de Estrategias de Gestión de las Microempresas. Como alternativa de solución a esta problemática se plantea la utilización de las TIC y Gobierno electrónico en el desarrollo local de las municipalidades.

En relación a la Supervisión. Según (Flórez, 2015), indica que el grado de supervisión en las acciones de control logra el progreso humano y económico. Por consiguiente, se identifica que influye en la administración del gobierno local del municipio. De acuerdo con (Samaniego, 2013), señala que el monitoreo o supervisión influyen en la mejora de procedimientos, y es muy importante para el mejoramiento constante, si se supervisan y controlan, realizaran un mejor trabajo, teniendo en cuenta no cometer errores. Así mismo, (Zamata, 2016), señala que el elemento de Supervisión mínimamente debe obtener un grado de implementación para la valoración del estado de establecimiento y ejecución del SCl (Sistema de Control Interno) para la consecución de objetivos de la institución en los municipios. Además (Marin, 2016), recomienda, tener un sistema de implantación que cumpla el rol de elemento básico y de guía, teniendo que realizar el orden de las actividades, la determinación de la supervisión y cronograma del logro de metas; y que participe como un elemento de coordinación que logre los compromisos del personal administrativo y la alta dirección de la institución. 


\section{Materiales y métodos}

Journal of the Academy Número 1 Julio-Diciembre 2019 - Pag. 35

La investigación ha sido realizada en la Universidad Andina del Cusco, sede Sicuani, provincia de Canchis, la cual conforma el departamento del Cusco del Perú. Tiene por limite en el norte con la provincia de Quispicanchis, al este con Puno y al sur con Canas y al oeste con Acomayo y se ubica en la cuenca alta del rio Vilcanota, teniendo una superficie de $3999.27 \mathrm{~km}^{2}$. Su ubicación esta entre las coordenadas $14^{\circ} 30^{\prime} y$ $14^{\circ} 56^{\prime}$ de latitud sur y $71^{\circ} 24^{\prime}$ y $71^{\circ} 39^{\prime}$ de longitud oeste y altitud promedio $3548 \mathrm{msnm}$, está integrada por distritos de Pitumarca, Checacupe, Combapata, Tinta, San Pedro, San Pablo, Sicuani y Marangani, el clima de la provincia de Canchis es seco, se consideró una población de 240 funcionarios de las ocho municipalidades de todos los distritos de la provincia de Canchis. La investigación es importante puesto que determina la categoría de relación entre el desarrollo local de las municipalidades de Canchis y el control interno. Los objetivos específicos fueron determinar la correlación entre las dimensiones, supervisión, comunicación e información, acciones de control, valoración de riesgos, ambiente de control, y desarrollo local de los concejos municipales de la provincia de Canchis, determinándose posteriormente que si existe correlación entre estas variables. Los materiales e instrumentos utilizados fueron la escala de Likert. El alcance del estudio fue correlacional, el diseño considerado en la investigación por sus características particulares es de los no experimentales correspondiente a los de tipo descriptivo correlacional. En el tratamiento estadístico se utilizó la estadística inferencial, los datos cuantitativos se procesaron con el software estadístico SPSS.V24 y se aplicó el estadístico de factor de relación lineal no paramétrico de Spearman.

\section{Resultados y discusión}

Este trabajo de investigación, de acuerdo a sus peculiaridades, trata de la correlación en medio del control interno, sus componentes y el desarrollo local de las municipalidades de la provincia de Canchis.

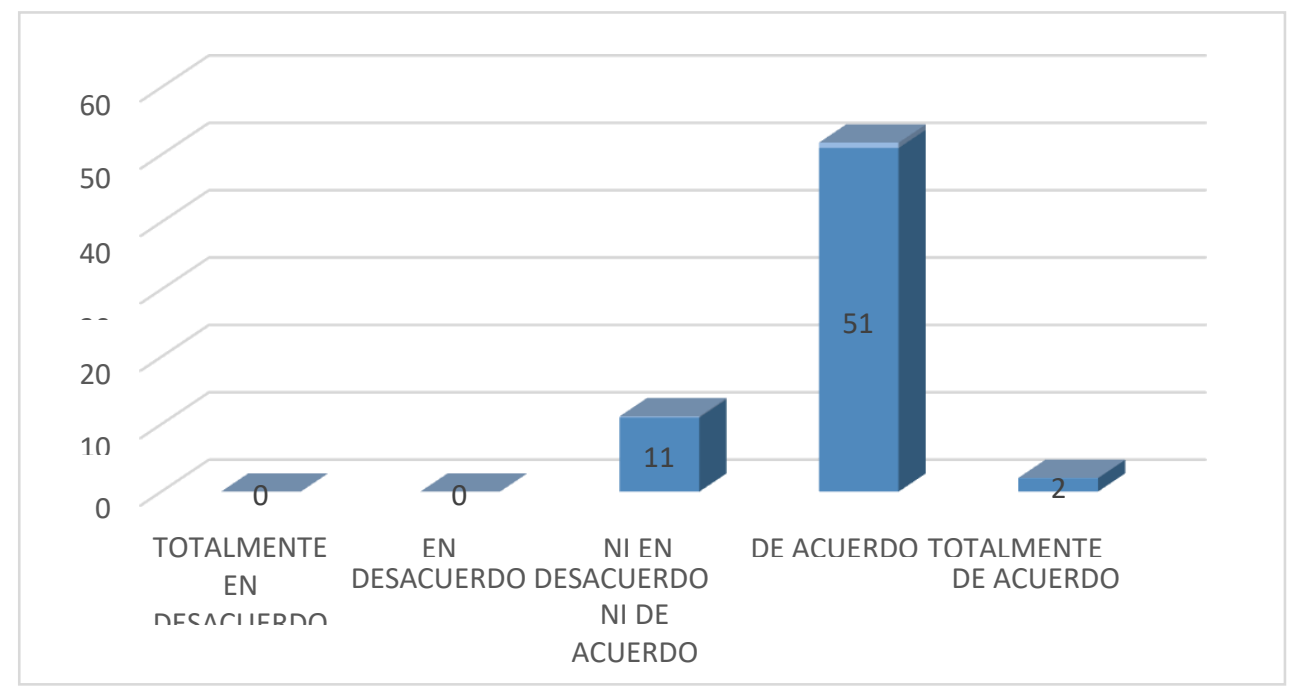

Grafico1. Correlación entre Control Interno y el Desarrollo Local de los Concejos Municipales de la provincia de Canchis 2018.

De un total de 64 funcionarios encuestados, 51 opinan estar de acuerdo respecto a la correlación entre control interno y el desarrollo local de los municipios de la provincia de Canchis, lo que equivale al $80 \%$ del total, 11 opinan estar ni en desacuerdo ni de acuerdo respecto a la relación entre control interno y desarrollo local de las municipalidades de la provincia de Canchis, lo que equivale al $17 \%$ del total, 2 opinan estar totalmente de acuerdo respecto a la relación entre control interno y desarrollo local de los concejos municipales de la provincia de Canchis, lo que equivale al $3 \%$ del total de funcionarios encuestados. Formalmente la prueba estadística es. Prueba de hipótesis general: 
Hipótesis:

Journal of the Academy Número 1 Julio-Diciembre 2019 - Pag. 36

Tabla 1. Correlación entre Control interno y Desarrollo Local de los concejos municipales de la provincia de Canchis 2018.

\begin{tabular}{|c|c|c|c|c|}
\hline & & & $\begin{array}{l}\text { Control } \\
\text { Interno }\end{array}$ & $\begin{array}{l}\text { Desarrollo } \\
\text { Local }\end{array}$ \\
\hline \multirow[t]{6}{*}{ Rho de Spearman } & $\begin{array}{l}\text { Control } \\
\text { Interno }\end{array}$ & $\begin{array}{l}\text { Coeficiente de } \\
\text { correlación }\end{array}$ & 1,000 &, $910^{* *}$ \\
\hline & & Sig. (bilateral) & & 0,000 \\
\hline & & $\mathrm{N}$ & 64 & 64 \\
\hline & $\begin{array}{l}\text { Desarrollo } \\
\text { Local }\end{array}$ & $\begin{array}{l}\text { Coeficiente de } \\
\text { correlación }\end{array}$ & $910^{* *}$ & 1,000 \\
\hline & & Sig. (bilateral) & 0,000 & \\
\hline & & $\mathrm{N}$ & 64 & 64 \\
\hline
\end{tabular}

De aquí Rho de Spearman $=0,910$ y Sig $=0,000$

Como: $\mathrm{P}$ value $=\mathrm{Sig}=0,000<\alpha=0,05$, entonces se decide rechazar $\mathrm{H}_{0}$.

$\mathrm{H}_{0}=$ No existe correlación significante entre el control interno y desarrollo local en los concejos

municipales de la provincia de Canchis 2018.

$\mathrm{H}_{\mathrm{A}}=$ Existe correlación significativa entre el control interno y desarrollo local en los concejos

municipales de la provincia de Canchis 2018.

Nivel de significancia: $\alpha=0,05=5 \%$

Valor de prueba: Se realizó con la ayuda del Software SPSS. V24

Al respecto, ( Dugarte, 2012), añade que todos los elementos del control interno conforme a lo planteado por la crónica COSO son elementales para el establecimiento de patrones de control interno de la administración en la realización de trabajos puesto que determinan un entorno integral de control mediante la interacción de todos los elementos, implica una relación puesto que los controles se insertan a las acciones operativas como una estructura cuyo efecto se aplica al integrarse a los objetivos de los medios de la gestión pública del municipio. Por otro lado, (Guevara, 2017), sugiere que, los empleados públicos del municipio dirigidos por su alcalde, implementen el sistema de control interno conforme a lo establecido en las reglas internacionales (Informe COSO) y las reglas nacionales (Contraloría General de la República).

Además, (Crisologo, 2013), establece que el Control Interno incide en la Gestión de los Gobiernos Locales de las Municipalidades. También (Acuña, 2013) sostiene que el control interno incide de forma positiva en la Unidad de abastecimiento del municipio. Por otro lado, (Condo, 2017), concluye que se ha demostrado las causas de la relativa implementación del control interno, y estas son: el desconocimiento de algunos empleados públicos y trabajadores a la Implantación del Sistema de Control Interno; ausencia de actualización a los empleados públicos y trabajadores y personal idóneo en el tema; relativa importancia por parte de la gestión a la Implantación del Sistema de Control Interno y ausencia de disposición de recursos económicos para su implantación. Por otra parte, (Martínez, 2016), concluye que los controles internos aplicados a la Dirección de proyectos son eficientes porque permiten desarrollar los proyectos en tiempo y forma según lo planificado y con su debido seguimiento.

Además, es necesario considerar la conclusión a que llega (Melgarejo, 2016), en su trabajo de investigación, en la cual deduce que hay correlación significativa entre el Control interno y la Dirección administrativa del municipio. Contrariamente a lo anterior (Acuña, 2013), en su trabajo de investigación titulado Control Interno en el Área de abastecimiento de la Municipalidad de Cajas, concluye que el 
control interno es frágil y no adecuado en el Área, Departamento y Unidad de Abastecimiento de los Municipios, de igual manera se evidencio la situación actual de las organizaciones puesto que las revisiones definieron que los empleados públicos y los trabajadores no conocen las normas de control interno están en relación con el área de abastecimiento, por lo tanto el control interno incide de forma positiva en el área estudiada. Similarmente, (Ñañez, 2017), en su trabajo de investigación titulado Las Municipalidades del Perú: caso de la municipalidad provincial de Yauyos - Lima, 2017, concluye que muchos municipios peruanos y el municipio Provincial de Yauyos, carece del funcionamiento de un sistema de control interno adecuado, por lo que serían afectados y perjudicados los usuarios, la ciudadanía en general y la gestión del titular; además los recursos y bienes de la entidad no son utilizados adecuadamente por el incumplimiento de las normativas establecidas por la CGR por ello existe deficiencia en los servicios públicos. En forma similar, (Mamani, 2018), en su trabajo de investigación denominado Control Interno en el Área de Administración de la Municipalidad distrital de Huatasani de la provincia de Huancané, periodo 2015, concluye que el municipio distrital de Huatasani no está desarrollando la mayoría de los sub elementos y elementos del control interno definidos en el informe COSO, por consiguiente, el control interno no está incidiendo en forma positiva en la institución. Prueba de hipótesis especifica1:

Hipótesis:

$\mathrm{H}_{0}=$ No existe relación significativa entre ambiente de control y desarrollo local en los municipios de la provincia de Canchis 2018

$\mathrm{H}_{\mathrm{A}}=$ Existe relación significativa entre ambiente de control y desarrollo local en los municipios de la provincia de Canchis 2018.

Nivel de significancia: $\alpha=0,05=5 \%$

Valor de Prueba: Se realizó con la ayuda del software SPSS. V24

Tabla 2. Correlación entre Ambiente de Control y desarrollo Local de los municipios de la provincia de Canchis 2018

\begin{tabular}{cllll}
\hline & & & $\begin{array}{l}\text { Ambiente de } \\
\text { Control }\end{array}$ & $\begin{array}{l}\text { Desarrollo } \\
\text { Local }\end{array}$ \\
\hline Rho de Spearman & $\begin{array}{l}\text { Ambiente de } \\
\text { Control }\end{array}$ & $\begin{array}{l}\text { Coeficiente de } \\
\text { correlación }\end{array}$ & 1,000 &, $821^{* *}$ \\
& $\begin{array}{l}\text { Sig. (bilateral) } \\
\text { Desarrollo }\end{array}$ & $\begin{array}{l}\text { Coeficiente de } \\
\text { correlación }\end{array}$ &, $821^{* *}$ & 0,000 \\
& Local & Sig. (bilateral) & 0,000 & 64,000 \\
& & N & 64 & 64
\end{tabular}

**. La correlación es significativa en el nivel 0,01 (bilateral).

De aqui Rho de Spearman $=0,821$ y $\mathrm{Sig}=0,000$

Como: $\mathrm{P}$ value $=\mathrm{Sig}=0,000<\alpha=0,05$, entonces se decide rechazar $\mathrm{H}_{0}$.

El elemento ambiente de control establece un ambiente organizacional en favor a la práctica de, comportamientos, conductas y normas adecuadas, para concienciar a los integrantes de la institución e implicar una educación control interno. (Vizcarra, 2017). Respecto a este componente, Samaniego (2013), señala que el Ambiente de Control incide en la definición de metas y objetivos de la institución. 
Por otra parte (Soto 2018), sugiere que las municipalidades distritales deben contar con la existencia de documentos de gestión como evaluaciones de clima laboral; (Manrique, 2015), plantea al gerente de la municipalidad, corregir el aspecto correspondiente al componente del ambiente de control y se recomienda a la autoridad y al gerente de la Municipalidad a mejorar la valoración de la estructura del control interno, con el fin de detectar y corregir sus defectos y mejorar constantemente sus reglas de control interno para que se apliquen a las acciones y relaciones de la entidad y sus dependencias. De forma contraria a lo anterior, (Guevara, 2017), concluye que los Municipios del Perú no aplican efectiva y eficientemente el sistema de control interno teniendo como causa a que en los municipios no hay existe un ambiente de control apropiado, los ROF Y MOF son desactualizados, lo que implica que los municipios no usen integralmente los recursos humanos y físico de que tienen, lo que implica el incumplimiento de los fines de la institución; lo cual en forma paralela afecta negativamente e ineficiente la aplicación y logro de los servicios públicos que dan los municipios. Prueba de hipótesis especifica 2:

Hipótesis:

$\mathrm{H}_{0}=$ No existe relación significativa entre Evaluación de Riesgos y el Desarrollo Local de las municipalidades de la provincia de Canchis 2018.

$\mathrm{H}_{\mathrm{A}}=$ Existe relación significativa entre Evaluación de Riesgos y Desarrollo Local de las municipalidades de la provincia de Canchis 2018. .

Nivel de significancia: $\alpha=0,05=5 \%$

Valor de prueba: Se realizó con la ayuda del software SPSS. V24

Tabla 3. Correlación entre evaluación de Riesgos y Desarrollo Local de las municipalidades de la provincia de Canchis 2018.

\begin{tabular}{lllll}
\hline & & & $\begin{array}{l}\text { Evaluación de } \\
\text { Riesgos }\end{array}$ & $\begin{array}{l}\text { Desarrollo } \\
\text { Local }\end{array}$ \\
\hline Rho de Spearman & $\begin{array}{l}\text { Evaluación de } \\
\text { Riesgos }\end{array}$ & $\begin{array}{l}\text { Coeficiente de } \\
\text { correlación } \\
\text { Sig. (bilateral) }\end{array}$ & 1,000 &, $910^{+*}$ \\
& $\mathrm{~N}$ & 64 & 0,000 \\
& $\begin{array}{l}\text { Desarrollo } \\
\text { Local }\end{array}$ & $\begin{array}{l}\text { Coeficiente de } \\
\text { correlación }\end{array}$ &, $910^{* *}$ & 64 \\
& Sig. (bilateral) & 0,000 & 1,000 \\
& $\mathrm{~N}$ & 64 & 64 \\
\hline **. La correlación es significativa en el nivel 0,01 (bilateral). & \\
\hline
\end{tabular}

De aquí Rho de Spearman $=0,910$ y Sig $=0,000$

Como: $\mathrm{P}$ value $=\mathrm{Sig}=0,000<\alpha=0,05$, entonces se decide rechazar $\mathrm{H}_{0}$.

En relación a esta componente, ( Boza, et al. , 2013), propone la implementación de un Sistema de Gestión en salud, una guía que oriente su aplicación, y una herramienta que favorezca su evaluación dentro de la Municipalidad.

Así mismo se establece que el grado de valoración de contingencia del control interno que incide es significante en el mejoramiento de condición de vida de los ciudadanos (Florez, 2015); por otra parte, (Crisologo, 2013), deduce que el nivel de evaluación de riesgo influye en el mejoramiento de la calidad de vida de los habitantes. Además, (Ñañez, 2017), sugiere identificar y analizar los riesgos que pueda perjudicar a la entidad; a la vez realizar acciones de supervisión para prevenir errores, lograr objetivos adecuados y para el bien común. Prueba de hipótesis especifica 3: 
Hipótesis:

$\mathrm{H}_{0}=$ No existe relación significativa entre Actividades de Control y Desarrollo Local de las municipalidades de la provincia de Canchis 2018.

$\mathrm{H}_{\mathrm{A}}=$ Existe relación significativa entre Actividades de Control y Desarrollo Local de los municipios de la provincia de Canchis 2018.

Nivel de significancia: $\alpha=0,05=5 \%$

Valor de prueba: Se realizó con la ayuda del software SPSS. V24

Tabla 4. Correlación entre Actividades de Control y Desarrollo Local de los concejos municipales de la provincia de Canchis 2018.

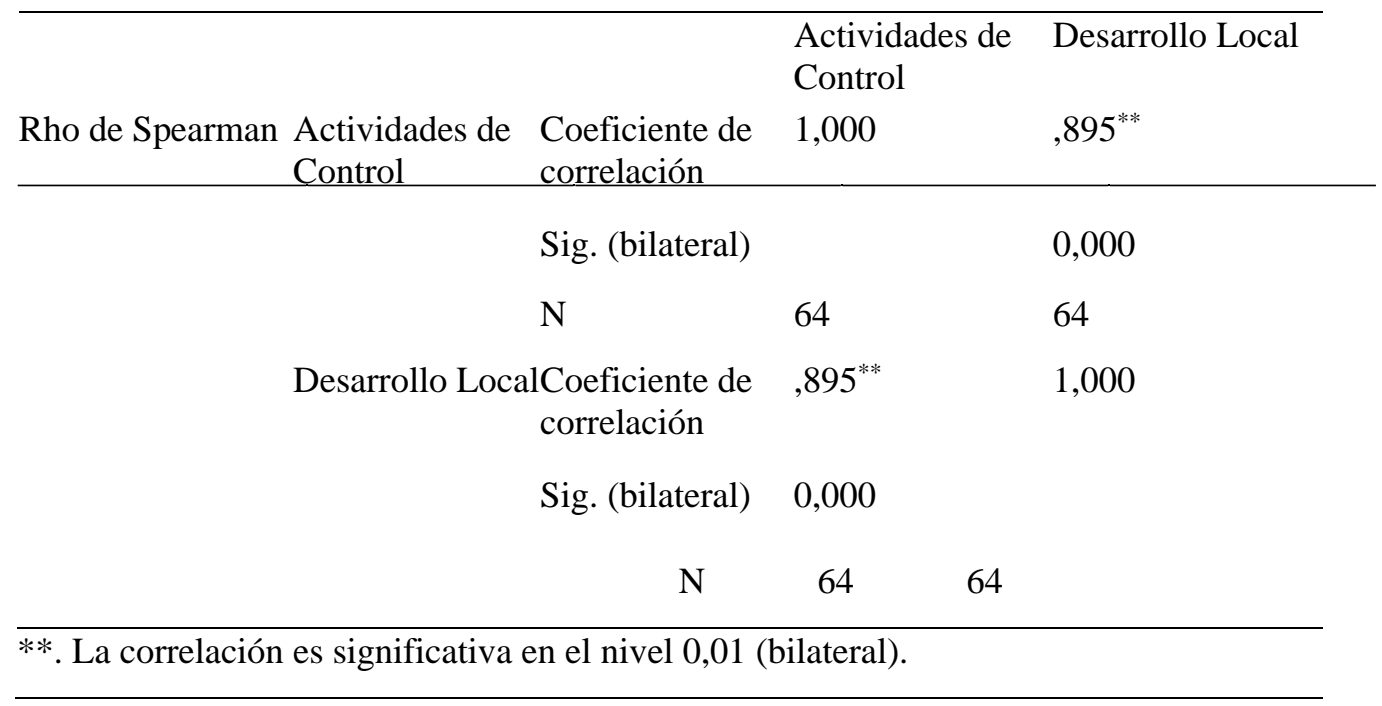

De aquí Rho de Spearman $=0,895$ y Sig $=0,000$

Como: $P$ value $=\mathrm{Sig}=0,000<\alpha=0,05$, entonces se decide rechazar $\mathrm{H}_{0}$.

En relación a esta componente, (Florez, 2015), establece que los grados de acciones de control inciden en el rédito del presupuesto de gasto versus la realización presupuestal. Además (Acuña, 2013), señala que el Sistema de Control Interno mejora de manera efectiva en las acciones de control de bienes patrimoniales y de almacén del municipio.

Por otra parte, (Layme, 2014), señala que entre reglas y procesos de control interno, se tiene significancia estadística, o sea se propone las reglas y procesos básicos para la ejecución del Sistema de Control Interno que optimicen sus acciones y relaciones, en la SPBS (Subgerencia de personal y Bienestar Social). Prueba de hipótesis especifica 4:

Hipótesis:

$\mathrm{H}_{0}=$ No existe relación significativa entre Comunicación e Información y Desarrollo Local en los concejos municipales de la provincia de Canchis 2018.

$\mathrm{H}_{\mathrm{A}}=$ Existe relación significativa entre Información y Comunicación y Desarrollo Local en las municipalidades de la provincia de Canchis 2018.

Nivel de significancia: $\alpha=0,05=5 \%$

Valor de prueba: Se realizó con la ayuda del software SPSS24. V24

Tabla 5. Correlación entre Comunicación e Información y Desarrollo local de los concejos municipales de la provincia de Canchis 2018. 


\begin{tabular}{|c|c|c|c|c|}
\hline & & & $\begin{array}{l}\text { Información y } \\
\text { Comunicación }\end{array}$ & Desarrollo Local \\
\hline \multirow[t]{6}{*}{ Rho de Spearman } & \multirow[t]{3}{*}{$\begin{array}{l}\text { Información y } \\
\text { Comunicación }\end{array}$} & $\begin{array}{l}\text { Coeficiente de } \\
\text { correlación }\end{array}$ & 1,000 &, $895^{* *}$ \\
\hline & & Sig. (bilateral) & & 0,000 \\
\hline & & $\mathrm{N}$ & 64 & 64 \\
\hline & \multicolumn{2}{|c|}{$\begin{array}{c}\text { Desarrollo Local Coeficiente de } \\
\text { correlación }\end{array}$} &, $895^{* *}$ & 1,000 \\
\hline & & Sig. (bilateral) & 0,000 & \\
\hline & & $\mathrm{N}$ & 64 & 64 \\
\hline
\end{tabular}

De aquí Rho de Spearman $=0,895$ y Sig $=0,000$

Como: $\mathrm{P}$ value $=\mathrm{Sig}=0,000<\alpha=0,05$, entonces se decide rechazar $\mathrm{H}_{0}$.

Teniendo en cuenta este componente, la comunicación municipal considera a la gestión local en su conjunto: sus áreas, estrategias, proyectos, fines, etc. Según (Florez, 2015), determina que el nivel de comunicación e información mejora en forma favorable los grados de eficiencia, eficacia y economía. 0 sea hay una incidencia significante referente a la administración del gobierno local de las municipalidades. Similarmente (Crisologo 2013), corrobora que el nivel de comunicación e información mejora de forma favorable los grados de eficiencia, eficacia y economía. Por otra parte según Zamata (2016), una de las funciones del CCl es comunicar a todo el personal de la importancia de contar con un SCl. Por otro lado, según (Samaniego, 2013), la Comunicación y la Información si inciden en la definición de Tácticas de Gestión de las Microempresas. Tratándose de un tema importante se plantea como alternativa de solución la utilización de las TIC y Gobierno electrónico en el control interno y el desarrollo local de las municipalidades. Prueba de hipótesis especifica 5:

Hipótesis:

$\mathrm{H}_{0}=$ No existe relación significante entre Supervisión y Desarrollo Local de las municipalidades de la provincia de Canchis 2018.

$\mathrm{H}_{\mathrm{A}}=$ Existe relación significativa entre Supervisión y Desarrollo Local en las municipalidades de la provincia de Canchis 2018.

Nivel de significancia: $\alpha=0,05=5 \%$

Valor de prueba: Se realizó con la ayuda del Software SPSS24. V24

Tabla 6. Correlación entre Supervisión y Desarrollo Local de los concejos municipales de la provincia de Canchis 2018. 


\begin{tabular}{|c|c|c|c|c|}
\hline & & & $\begin{array}{l}\text { Información y } \\
\text { Comunicación }\end{array}$ & Desarrollo Local \\
\hline \multirow[t]{6}{*}{ Rho de Spearman } & $\begin{array}{l}\text { Información y } \\
\text { Comunicación }\end{array}$ & $\begin{array}{l}\text { Coeficiente de } \\
\text { correlación }\end{array}$ & 1,000 &, $821^{* *}$ \\
\hline & & Sig. (bilateral) & & 0,000 \\
\hline & & $\mathrm{N}$ & 64 & 64 \\
\hline & Desarrollo Loca & $\begin{array}{l}\text { Coeficiente de } \\
\text { correlación }\end{array}$ &, $821^{* *}$ & 1,000 \\
\hline & & Sig. (bilateral) & 0,000 & \\
\hline & & $\mathrm{N}$ & 64 & 64 \\
\hline
\end{tabular}

De aquí Rho de Spearman $=0,821$ y $\operatorname{Sig}=0,000$

Como: $P$ value $=\operatorname{Sig}=0,000<\alpha=0,05$, entonces se decide rechazar $\mathrm{H}_{0}$.

Tratándose de esta componente, según (Flórez, 2015), indica que el grado de supervisión en las acciones de control logra crecimiento humano y económico. Identificándose que incide en la administración del gobierno local del municipio. De acuerdo con (Samaniego, 2013), señala que la Supervisión o Monitoreo influyen en la mejora de procedimientos, y es de mucha importancia para la mejora constante. Así mismo, (Zamata, 2016), señala respecto al elemento Supervisión mínimamente debe obtener un grado de implementación para la valoración del nivel de implementación y ejecución del sistema de control interno de tal manera que se logren los objetivos institucionales en los municipios. Marín, R.M. (2015), recomienda, premunirse de un plan de implantación que sirva de elemento guía para el orden de las actividades, la determinación del programa y seguimiento de la ejecución de objetivos y metas. Contradictoriamente a lo anterior, (Soto, 2018), en su tesis titulada, Diagnostico del Ambiente de Control Interno en la Municipalidad distrital de Jesús-Cajamarca, 2017, concluye que la gerencia establece un nivel de cumplimiento medio respecto a la supervisión de la alta dirección, las estructuras, las líneas de reporte y los niveles de autoridad y responsabilidad apropiadas para la consecución de los objetivos en la municipalidad.

\section{Conclusiones}

Existe relación lineal muy alta o muy fuerte significativa entre el Control Interno y el Desarrollo Local de los municipios de la provincia de Canchis. Análogamente existe relación lineal muy alta significativa entre los componentes del Control Interno y Desarrollo Local de las municipalidades de la provincia de Canchis respectivamente.

\section{Referencias bibliográficas}

Acuña, J. (2013), Control interno en el área de abastecimiento de la municipalidad distrital de San Agustín de Cajas. Tesis para optar el título de Contador Público, Universidad del Centro del Perú. Huancayo, Perú. Retrieved from http://repositorio.uncp.edu.pe/handle/UNCP/2423

Boza et al. (2013), Propuesta para el desarrollo de un sistema de gestión en salud en el gobierno local: experiencia de la municipalidad de Tiba. Tesis de Licenciatura, Universidad de Costa Rica. Costa Rica. Retrieved from https://www.google.com/search?ei=I39HXIfyAcXAsQX 
Condo, E. N. (2017), El Control interno en el proceso de ejecución presupuestal de la municipalidad distrital de Santa Rosa Melgar Puno, periodo 2012-2013. Tesis para optar el título profesional de Contador Público. Universidad Nacional del Altiplano. Puno, Perú. Retrieved from http://repositorio.unap.edu.pe/handle/UNAP/5254

Crisologo, M. F. (2013). Control interno en la gestión de los gobiernos locales del callejón de HuaylasAncash. Tesis para optar el grado académico de Maestría en Contabilidad y Finanzas, con mención en Auditoría y Control de Gestión Empresarial. Universidad San Martin de Porres. Lima, Perú. Retrieved from http://www.repositorioacademico.usmp.edu.pe/bitstream/usmp/639/3/crisologo_mf.pdf

Dugarte, J. C. (2012). Estándares de control interno administrativo en la ejecución de obras civiles de los órganos de la administración pública municipal. Tesis presentada como requisito para optar al grado de Magister en Ciencias Contables, Universidad de los Ángeles. Mérida, Venezuela. Retrieved from http://pcc.faces.ula.ve/Tesis/Maestria/Jose Candelario Dugarte Rodríguez/Tesis José Candelario.pdf

Flórez, E. (2015). Repercusión del Control Interno en la Gestión Municipal de la Municipalidad provincial de San Román. Tesis para optar el Grado Académico de Magister en Administración en la Universidad Andina Nestor Cáceres Velásquez. Perú. Retrieved from http://repositorio.uancv.edu.pe/handle/UANCV/341

Guevara, Y. (2016). Caracterización del control interno de las municipalidades del Perú: caso municipalidad provincial de Moyobamba, 2015. Tesis de licenciatura, Universidad Católica Los Ángeles Chimbote. Perú. Retrieved from http://repositorio.uladech.edu.pe/handle/123456789/1990

Hurtado de la Cruz, G. (2015). Control interno en la gestión de obras por administración directa de la municipalidad distrital de San Juan de Lurigancho-2013. Tesis para optar el título profesional de Contador Público. Universidad Nacional de Huancavelica. Perú. Retrieved from http://repositorio.unh.edu.pe/bitstream/handle/UNH/345/TP - $\quad$ UNH. 0057.pdf?sequence=1\&isAllowed=y

Layme, E. S. (2014). Evaluación de los componentes del sistema de control interno y sus efectos en la gestión de la subgerencia de personal y bienestar social de la municipalidad provincial Mariscal Nieto, 2014. Tesis para obtener el título profesional de Contador Público. Un. Moquegua, Perú. Retrieved from http://repositorio.ujcm.edu.pe/handle/ujcm/22

Mamani, E. (2017). Control interno en el área de administración de la municipalidad distrital de Huatasani de la provincia de Huancané, periodo 2018. Tesis para optar el título profesional de contador público, Universidad Católica Los Ángeles Chimbote. Perú.

Retrieved from http://repositorio.uladech.edu.pe/handle/123456789/2859

Manrique, J. (2018). El control interno y su influencia en la gestión administrativa de las Municipalidades del Perú: caso municipalidad distrital de Nuevo Imperial, 2015. Tesis para optar el título profesional de Contador Público. Universidad católica Los Ángeles Chimbote. Perú. Retrieved from http://repositorio.uladech.edu.pe/handle/123456789/2863

Marin, R. M. (2016). Gestión del sistema de control interno en la toma de decisiones de las municipalidades distritales de la provincia de Puno 2015. Tesis para optar el grado académico de Magister en Administración, Mención: Gerencia y Control de Gobiernos Locales y Regional. Peru. Retrieved from http://repositorio.uancv.edu.pe/handle/UANCV/445 
Martínez, M. y Del Carmen, M. (2016). Análisis del Control Interno y su Aplicación en la dirección de Proyectos de la Alcaldía Municipal de Matagalpa (Almat), primer Semestre 2015. Tesis de Maestría, Universidad Nacional Autónoma de Nicaragua. Managua, Nicaragua. Retrieved from http://repositorio.unan.edu.ni/1790/

Melgarejo, N. P. (2016). Control interno y gestión administrativa según trabajadores de la municipalidad provincial de Chota 2016. Tesis para optar el grado de Maestra En Gestión Pública. Universidad Cesar Vallejo. Perú. Retrieved from http://repositorio.ucv.edu.pe/handle/UCV/9033

Ñañez, O. E. (2017). Caracterización del control interno de las municipalidades del Perú: caso de la municipalidad provincial de Yauyos -Lima, 2017. Tesis para optar el título profesional de Contador Público, Universidad Católica Los Ángeles de Chimbote. Perú.

Retrieved from http://repositorio.uladech.edu.pe/handle/123456789/1855

Samaniego, C. M. (2013). Incidencias del Control Interno en la optimización de la gestión de las microempresas en el distrito de Chaclacayo. Tesis para optar el Grado académico de Maestro en Auditoría y Control de Gestión Empresarial, Universidad San Martin de Porres. Perú. Retrieved from http://www.repositorioacademico.usmp.edu.pe/bitstream/usmp/645/3/samaniego_cm.pdf

Soto, E. (2017). Diagnóstico del ambiente de control interno en la municipalidad distrital de JesúsCajamarca, 2017.Tesis para optar el título profesional de: Contador Público, Universidad Nacional de Cajamarca. Perú. Retrieved from http://repositorio.unc.edu.pe/handle/UNC/1889

Vizcarra, M. Y. (2017). El Control Interno y su incidencia en la gestión administrativa de la municipalidad provincial de Mariscal Nieto-2015. Tesis para optar el título profesional de Contador Público, Universidad José Carlos Mariátegui. Moquegua, Perú. Retrieved from http://repositorio.ujcm.edu.pe/handle/ujcm/140

Zamata, R. M. (2016). Evaluación del estado de implementación y aplicación del sistema de control interno para el logro de objetivos institucionales en la municipalidad provincial de San Román en el periodo 2014. Tesis de licenciatura, Universidad Nacional del Altiplano. Puno, Perú. Retrieved from http://repositorio.unap.edu.pe/handle/UNAP/1806 